\title{
Flaky Test Detection in Android via Event Order Exploration
}

\author{
Zhen Dong \\ National University of Singapore, Singapore \\ zhen.dong@comp.nus.edu.sg \\ Xiao Liang Yu \\ National University of Singapore, Singapore \\ xiaoly@comp.nus.edu.sg
}

\author{
Abhishek Tiwari \\ National University of Singapore, Singapore \\ dcsabhi@nus.edu.sg \\ Abhik Roychoudhury \\ National University of Singapore, Singapore \\ abhik@comp.nus.edu.sg
}

\begin{abstract}
Validation of Android apps via testing is difficult owing to the presence of flaky tests. Due to non-deterministic execution environments, a sequence of events (a test) may lead to success or failure in unpredictable ways. In this work, we present an approach and tool FlakeScanner for detecting flaky tests through exploration of event orders. Our key observation is that for a test in a mobile app, there is a testing framework thread which creates the test events, a main User-Interface (UI) thread processing these events, and there may be several other background threads running asynchronously. For any event $e$ whose execution involves potential non-determinism, we localize the earliest (latest) event after (before) which $e$ must happen. We then efficiently explore the schedules between the upper/lower bound events while grouping events within a single statement, to find whether the test outcome is flaky. We also create a suite of subject programs called FlakyAppRepo (containing 33 widely-used Android projects) to study flaky tests in Android apps. Our experiments on the subject-suite FlakyAppRepo show FlakeScanner detected 45 out of 52 known flaky tests as well as 245 previously unknown flaky tests among 1444 tests.
\end{abstract}

\section{CCS CONCEPTS}

- Software and its engineering $\rightarrow$ Software testing and debugging;

\section{KEYWORDS}

flaky tests, non-determinism, concurrency, event order

\section{ACM Reference Format:}

Zhen Dong, Abhishek Tiwari, Xiao Liang Yu, and Abhik Roychoudhury. 2021. Flaky Test Detection in Android via Event Order Exploration. In Proceedings of the 29th ACM foint European Software Engineering Conference and Symposium on the Foundations of Software Engineering (ESEC/FSE '21), August 23-28, 2021, Athens, Greece. ACM, New York, NY, USA, 12 pages. https://doi.org/10.1145/3468264.3468584

\section{INTRODUCTION}

Regression testing aims to discover the adverse effects of the recently added code changes. Ideally, a test failure during regression

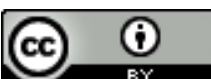

This work is licensed under a Creative Commons Attribution International 4.0 License. ESEC/FSE '21, August 23-28, 2021, Athens, Greece

(C) 2021 Copyright held by the owner/author(s).

ACM ISBN 978-1-4503-8562-6/21/08.

https://doi.org/10.1145/3468264.3468584 testing should reliably signal issues with recent code modifications. However, some test failures are not due to the recent updates but due to flaky tests. Recent research $[6,17,30]$ establishes flaky tests as tests that non-deterministically pass or fail when running on the same code version. Unfortunately, the presence of flaky tests significantly harms developers' productivity $[1,25,30]$.

Android is a reactive system, and its non-deterministic execution environment often causes concurrency-related flaky tests. In Android, a GUI test usually simulates user interactions to exercise the app's functionality. However, due to non-determinism, a test could generate such interactions at "incorrect" timings. For example, a test to download an image and then open it without considering the internet's speed could show non-deterministic behavior. A potential reason for such concurrency issues is the lack of synchronization among threads, e.g., in the failing run, a background thread would still be downloading the image while the testing thread tries to open it without synchronization with the background thread.

Recent studies [34, 42] confirm that such synchronization issues lead to a significant number of flaky tests in Android apps. Thorve et al. [42] studied 77 flakiness-related commits in Android projects and discovered that $36 \%$ failures are due to synchronization issues between testing thread and app under test, e.g., a test accesses data in the app before the data is available. Similarly, Romano et al.'s study [34] shows that 33\% flaky test failures are caused by threads' synchronization issues (tests interacting with the UI elements before the elements are fully loaded).

Challenges. Exposing a concurrency-related flaky test in a test suite is challenging as such flakiness is only observed when events get executed in a particular order. In Android, a test's event execution order may show non-determinism due to the non-deterministic execution environment. Some of such (irregular) event orders may cause a test to fail/pass occasionally. Consequently, detecting such a situation would require exploring such event orders deterministically; unfortunately, this poses a set of challenges for the existing flaky test detection approaches, which we discuss below.

- RERUN. A typical way to detect a flaky test is to rerun (expressed as RERUN) it multiple times. If it passes in some runs and fails in others, the test is marked as flaky. However, this approach can struggle to detect concurrency-related flaky tests because the target's execution environment may not introduce the needed non-determinism. Besides, it may require too many runs to witness the flakiness.

- Noise Based. Several approaches [4, 38, 40, 48] run tests with environmental perturbations (e.g., changing CPU load or test execution orders) in the hope of observing unexpected behaviors. 
Despite being simple and easy to be adopted in practice, adding noise does not guarantee to explore different execution orders. Consequently, they fail to detect many concurrency related flaky tests, as observed during our experiments.

- Event Race. The concurrency-related flakiness may be caused due to a synchronization problem on the testing thread. Besides, a concurrency bug on the app under test could also cause a non-deterministic test failure. Thus, in principle, approaches identifying concurrency bugs should also detect a subset of such flaky tests. Recent works [8, 18, 19, 24, 32] leverage program analysis techniques to analyze possible races in Android apps. For instance, EventRacer [8] records the execution trace of a test and statically analyzes it to find potential races. However, such approaches are prone to multiple false positives, which may create more problems for the developers during the Continuous Integration $(\mathrm{CI})$ process.

Our Approach. We introduce a lightweight technique that automatically detects a concurrency flaky test in Android apps within few test runs. Our technique explores the possible event execution orders in a test by scheduling a non-deterministic (async) event such that each test run explores a different event execution order. Besides, we identify and explore event orders in which the test flakiness manifests such that a flaky test can be detected in a few test runs.

Insight. Possible event execution orders are introduced by nondeterministic execution of async events. Android apps use a concurrent event-driven model, in which only the main (UI) thread can access GUI and processes user events. To keep GUI responsive, the UI thread offloads a long-running task (e.g., internet accessing) to a background thread. Once the task is finished, the background thread sends an event (called async event) to the UI thread to perform a GUI update. In a test run, the testing thread and background threads send events to the UI thread simultaneously, resulting in races among the events; the test may pass for some event orders and fail in others. For the example mentioned earlier, the testing thread simulates the "open the image" event, and the background thread downloads the image and sends an update event after completing the download. A race may occur between these two events. If the internet is fast, the update event reaches the UI thread before the "open the image" event, and the test passes. Otherwise, the "open the image" event is processed first, and the test fails.

Event Order Exploration. Leveraging these insights, our approach explores possible event execution orders by scheduling an async event in a test run. Our approach first identifies the schedule space for each async event with dynamic analysis and schedules the async event in its schedule space to avoid infeasible orders. To manifest a flaky test failure quickly, our approach schedules events at positions where the test is more likely to fail. Specifically, it schedules an async event at a statement boundary position. The statement boundary position is between the last event that a test statement triggers and the first event that the next statement triggers. We note that the tests are often flaky due to missing an appropriate synchronization operation between two test statements, e.g., the synchronizing operation after clicking a download button. Thus we are more likely to trigger a test failure if an async event is executed after executing certain test statements.
Instrumentation-free Tool. We implement our approach into a tool called FlakeScanner, which leverages the debug mode supported in the Android framework to perform dynamic analysis based event scheduling. Thus FlakeScanner requires no instrumentation in either Android apps or the Android framework and works on both Android emulators and devices. According to the study [41], code instrumentation often disrupts test executions and prevents the manifestation of flaky test failures. Moreover, FlakeScanner supports multiple widely-used testing frameworks with which developers write tests such as Espresso [2] or Robotium [13].

Experiment. We evaluated FlakeScanner on 33 widely-used Android projects. Our experiments show that FlakeScanner successfully detected 45 out of 52 known flaky tests. On average, it detected a flaky test within three test runs. FlakeScanner outperformed the recently published flaky test detection technique Shaker [40] and the baseline tool RERUN in terms of both the number of detected flaky tests and average execution time. FlakeScanner also detected 245 flaky tests that were previously unknown. Out of these 245 unknown flaky tests, we reported 20 to developers; 13 out of these 20 have been confirmed and addressed by developers.

Contributions. Our contributions can be summarized as follows:

- We present an event scheduling approach that explores different event execution orders for each test run to detect concurrency-related flaky tests in Android apps. To avoid exploring all possible event orders, our approach adopts heuristics to identify and explore event orders in which the test flakiness is likely to occur.

- We implement our approach into an instrumentation-free tool that works on Android emulators and physical devices while supporting widely-used Android testing frameworks.

- We curate a suite of subject programs containing 33 widelyused Android projects with developer tests, called FlakyAppRepo for evaluating flaky test detection techniques. To facilitate future research on flaky tests, we make our prototype FlakeScanner and subject-suite FlakyAppRepo available at https://github.com/AndroidFlakyTest

\section{BACKGROUND}

Android Concurrency Model. Figure 1 depicts Android's eventdriven concurrency model. Each Android app has a main thread (also called UI thread); this thread processes events generated by users or the Android system. As shown in Figure 1, the UI thread maintains an event queue and an event looper. The queue is used to store received events, and the looper sequentially dequeues events from the queue and dispatches them to corresponding handlers for processing. Android adopts a single-UI-thread model in which only the main thread can access GUI objects. To achieve rapid UI responsiveness, the UI thread offloads long-running tasks such as network access to background threads, called async threads since they communicate with the UI thread asynchronously. Once tasks are completed, async threads post an event (called async event marked in blue in Figure 1) to the UI thread, and the UI thread updates the results to GUI. Event races may occur in this model. The UI thread and async threads run concurrently. The duration that an async thread will take to complete a task and post 


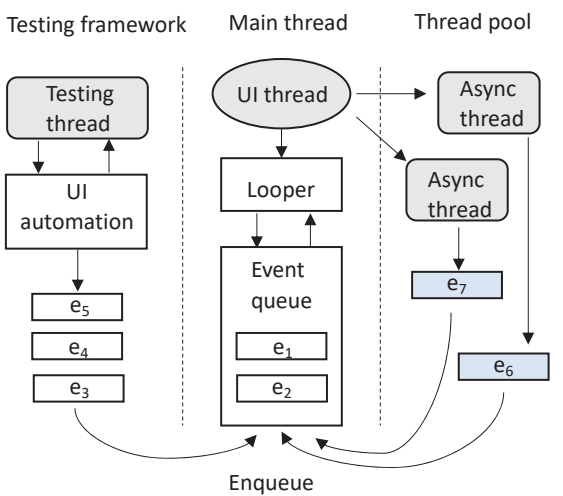

Figure 1: Android concurrency model \& testing framework.

an async event is non-deterministic, depending on the current execution environment. Thus, a race might occur among posted async events and others, leading to non-deterministic execution orders. In the example in Figure 1, there are multiple event orders which might occur in the execution, for instance, $\left\langle e_{1}, e_{2}, e_{6}, e_{3}, e_{4}, e_{5}, e_{7}\right\rangle$ and $\left\langle e_{1}, e_{2}, e_{3}, e_{4}, e_{5}, e_{6}, e_{7}\right\rangle$.

Instrumented Tests \& Testing Frameworks. Instrumented tests are tests that run on physical devices and emulators, and they can invoke the Android framework APIs to control app under test at runtime. These tests are often executed in a separate thread (called testing thread) to simulate user interactions, as shown in Figure 1. The Android system supports multiple testing frameworks to help developers write tests, e.g., Espresso [2]. To achieve reliable tests, these frameworks provide mechanisms to synchronize user interactions with app under test. For instance, when method onView() is invoked in a test, Espresso waits to perform the corresponding UI action or assertion until the event queue is empty, background threads are terminated, and user-defined resources are idle.

\section{A MOTIVATING EXAMPLE}

To set the stage for our event order exploration technique for flaky test detection, we first illustrate the characteristics of a flaky test and then exemplify current approaches' inadequacy through Listings 1-3. These listings show parts of CaptureLocationActivityTest, a flaky test taken from RapidPro Surveyor app. This test intends to validate whether the app can successfully obtain the location data using Google APIs. As shown in Listing 1, the test launches CaptureLocationActivity (Line 2), the activity to capture the location data (Listing 2). Later, the test emulates a button click to fetch the location at line 5 in Listing 1 , and validates whether the location is successfully fetched at line 7 in Listing 1.

Despite appearing straightforward, the test displays non-deterministic behavior, i.e., it is flaky. As explained earlier, the test runs in a testing thread, and the activity under test runs in the app's UI thread. The activity (CaptureLocationActivity) offloads fetching location data via Google API client to an async thread (Listing 3). After obtaining the location data, the async thread updates the result to the UI thread, and then the UI thread updates this result to CaptureLocationActivity. Due to the lack of synchronization between the testing thread and async thread, the async thread might send the location data before or after the testing thread validates it. If the validation occurs before the location data is received, the test fails, else it passes.

Detecting concurrency-related flaky tests is non-trivial as such failures manifest when the events are executed in a specific order. The traditional approach RERUN blindly executes the test many times in the hope of witnessing the flaky test failures. However, this approach becomes ineffective when the environment under which the test runs does not produce the required non-determinism. For example, RERUN failed to witness the flaky test failure in CaptureLocationActivityTest during our experiments. Another approach is to add noise in the execution environment to increase the likelihood of observing such errors. However, such approaches do not proactively explore different event execution orders and are prone to miss crucial event orderings. In our evaluations, the recent (noise-based) related work Shaker [40] failed to detect many confirmed flaky tests, including CaptureLocationActivityTest. We also evaluated EventRacer, a dynamic analysis based technique for detecting event races in Android apps. However, such approaches are prone to report many races. For example, in our evaluation, EventRacer reported over 200 data races for CaptureLocationActivityTest. Unfortunately, none of these reported data races from EventRacer involve events originated from the testing thread, and hence do not demonstrate the specific flakiness we are illustrating in this section. Besides, EventRacer does not validate whether reported races can cause the test failure.

\section{OVERVIEW OF OUR APPROACH}

This section describes the main components of our framework and lists the principles that make event exploration suitable for detecting concurrency-related flaky tests. First, the category of flaky tests should be well defined. It is acceptable to give up on all classes of flaky tests and focus on one; concurrency-related flaky tests. Second, using only one test run, we explore (all) possible scheduling space of an async event. Third, we execute these new schedulings to observe the flaky behavior, i.e., there exist at least two executions for which a test depicts different outcome (pass/fail).

\subsection{Basic Concepts}

Before diving into details of our approach, we define a miniature domain-specific syntax extended from [20] for an Android test.

$$
\begin{array}{lll}
\text { Test } & T & :==\vec{S} \\
\text { Statement } & S & ::=\operatorname{post}(\vec{e}) \mid \text { ASSERTIONS } \mid \text { sYNC } \mid \text { other } \\
\text { Event } & e & ::=\vec{M}_{\text {UIThread }}\left|\vec{M}_{\text {backgroundThread }}\right| \vec{M}_{O S} \\
\text { Synchronize } & \text { SYNC } & :=\operatorname{SYNC}(\vec{S}) \\
\text { Message } & M
\end{array}
$$

A test $T$ is composed of a series of program statements $S$. For concurrency-related flaky tests, we are interested in exploring the scheduling space of events, and thus for ease of representation, we categorize a test statement into four parts; statements posting an event, assertion statements, testing framework-specific synchronizing statements, and all other Android-specific statements. An event, denoted as $e$, is a message object created in the UI thread 
a map between them. According to this map, we identify anchor events for the test. Defining the lower bound of an async event is now straightforward. Intuitively, for a given statement, the lower bound of an async event would always be the anchor event of this statement, as the anchor event will always happen-before async event (Observation 1).

Localizing the upper bound event of an async event $e_{\text {async }}$ is more involved and requires identifying events that depend on $e_{\text {as ync }}$, i.e., events that occur only after $e_{\text {async }}$. Such event dependence is maintained by thread synchronization operations. For instance, the testing framework Espresso uses onView() operation to synchronize the testing thread and app under test. The testing thread waits until specific threads or resources are idle to ensure that a widget specified by onView() shows up on the screen. Formally, we define the upper bound of an async event as:

Definition 4.4 (Upper Bound Event). Let $T$ be a test containing a set of statements $\vec{S}$. We define $e_{j}$ as the upper bound event for an async event $e_{a s y n c}$, where $e_{j}$ is the first event that cannot happenbefore e async.

We propose a what-if analysis to localize the upper bound event of $e_{i}$. Specifically, after the test is launched, we hook the async thread that posts $e_{i}$ at runtime and suspend it, keeping other threads free. Meanwhile, we monitor the testing thread to check at which statement the testing thread stops and waits for the suspended thread to be completed. Suppose that the testing thread stops at a statement $s$ on executing the event $e_{j}$. We then deem operations in $s$ to depend on $e_{i}$, and these operations will not be executed until $e_{i}$ is processed. Thus, the anchor event $e_{j}$ triggered by $s$ is upper bound event of $e_{i}$. The idea behind the analysis is what-if it takes forever to complete the long-running task that corresponds $e_{i}$; operations in a test that depend on $e_{i}$ will not be executed due to thread synchronization, and those that do not depend on $e_{i}$ will be executed.

\subsection{Scheduling Events}

Next, we explore the scheduling strategies for an async event $e_{a s y n c}$. Given the lower and upper bound events of $e_{\text {async }}$, the anchor events that lie between this interval are identified. Then, $e_{a s y n c}$ is scheduled before each of these anchor events. To manifest flaky test failures as soon as possible, we prioritize positions closer to the upper bound event (maximizing the runtime of the async event), i.e., we first schedule $e_{a s y n c}$ just before its upper bound and then move towards its lower bound.

For two reasons, we only explore positions before anchor events in the scheduling space of an async event instead of all possible positions. First, for non-anchor events in the scheduling space, their execution orders may change from run to run. Using them as hooks for event scheduling may introduce infeasible orders. Second, anchor events are boundary events of test statements. Scheduling an async event before them is likely to trigger a flaky test failure.

\section{METHODOLOGY}

Figure 2 shows the workflow of our approach. For a given test, our framework performs a concrete execution to trace all the generated events. Next, a map between the statements in the test and their

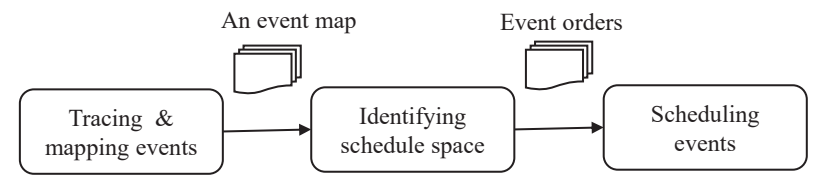

Figure 2: Workflow of exposing flaky tests through systematic schedule exploration.

corresponding events is created. The test is executed multiple times to compute possible schedules for async events. Consequently, a set of event orders that might occur in execution environments are created. Finally, we explore these possible event execution orders; if a test failure is detected during the exploration, the test is identified as a flaky test (since we have already seen passing runs of the test).

\subsection{Event Tracing and Mapping}

Event tracing is often used in the dynamic analysis of Android apps. It can be achieved by simply logging events that are generated at runtime. However, such techniques cannot fulfill our task. Event information (e.g., event id) produced in logs is dynamically generated by Android runtime and changes in each run. Our approach requires an event identifier to identify an event uniquely across different test runs. Async events that are identified during event tracing need to be hooked and scheduled in runs that are performed for event order exploration.

Event Identification. We identify an event based on interactions between the event and app under test at runtime. Two events triggered in different test runs are considered identical if: (1) they are triggered by the same test statement; (2) they are processed by the same sequence of methods at runtime. For instance, a pressDown event is associated with an identifier constructed using the line number of the statement that triggers the event and signatures of a sequence of methods that process it. This practice of event identification comes from our investigation of the Android framework.

Tracing and Mapping. Algorithm 1 outlines the procedure of event tracing and mapping. First, it launches the app under test and takes control of the Android runtime with a module called ARTHandler. Given a test $T$ consisting of a series of statements, ARTHandler runs the test $T$ in the testing thread and executes statements one by one (via the Android debugger). When one statement is executed, ARTHandlers monitors the event queue of the UI thread and hooks injected events. For each event, ARTHandler records the tuple $\left\langle i s A s y n c, s q_{m}\right\rangle$ where isAsync denotes whether it is an async event and $s q_{m}$ denotes the signatures of a sequence of methods that have processed the event. This tuple and the line number of the statement being executed form the event's identifier and get stored in a list (Line 11-14). As stated before, a statement in the test might perform long-running tasks, which are executed in async threads. When these tasks are completed is non-deterministic, and the async event might be posted after a long time. To not miss async events that are triggered by a single statement, we keep hooking events until two criteria are satisfied: (a) there are no new events and (b) the event queue of the UI thread is empty, which often indicates the system is not running tasks. This practice is also used in the 


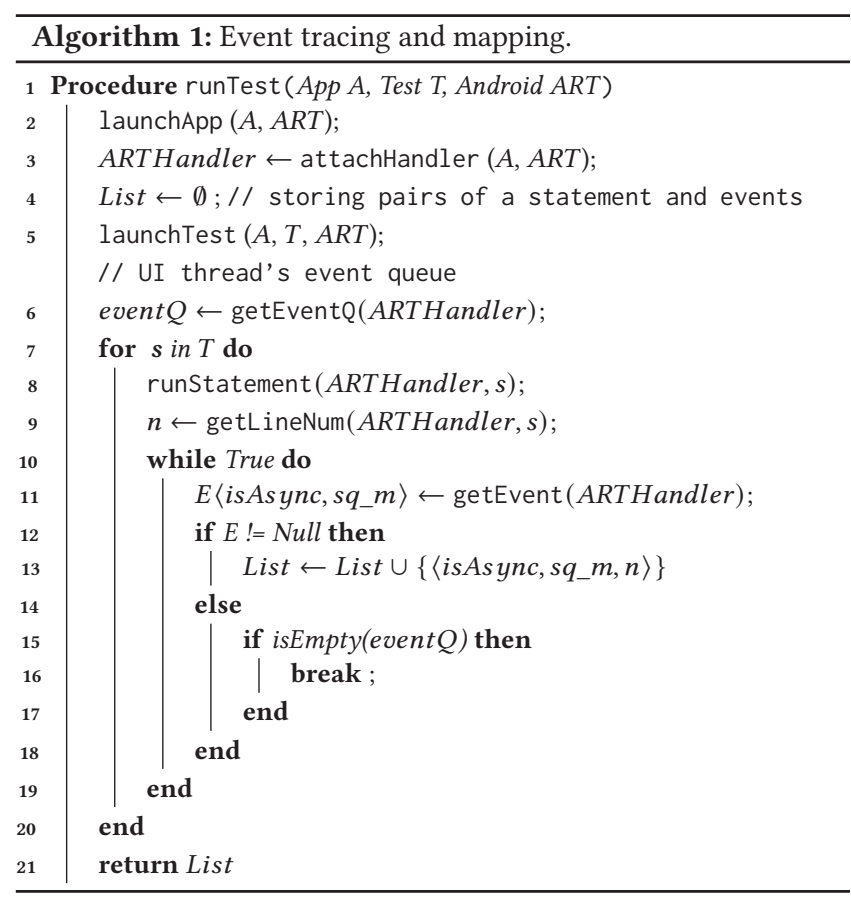

Espresso testing framework. Finally, a map between a statement and its events is returned via the List, and the first event of each statement is identified as an anchor event.

\subsection{Identifying Event Schedule Space}

To compute possible event execution orders, we perform a what-if dynamic analysis to resolve event dependencies caused by thread synchronization between apps and testing frameworks. Algorithm 2 shows the procedure of resolving event dependencies. It takes the event trace List generated in the previous step as input. For each async event $e_{\text {async }}$ in List, the algorithm launches the test and starts to hook event $e_{\text {async }}$ (Line 4-8). Once hooked, the algorithm suspends the thread that posts $e_{a s y n c}$ such that $e_{a s y n c}$ cannot be posted (Line 9). Meanwhile, it keeps checking the status of the testing thread (Line 10-14). If the testing thread's status is WAITING, it considers the testing thread is performing thread synchronization with threads in the app and waiting for $e_{a s y n c}$ to be executed. Thus, we consider the statement $s$ that is being executed in the testing thread attempts to trigger an event (e.g., $e_{j}$ ) which depends on $e_{\text {async. }}$. Therefore, the schedule space of $e_{a s y n c}$ is bounded by $e_{j}$, i.e., the first event that is triggered by $s$. So statement $s$ is identified as the upper bound of schedule space of async event $e_{\text {as ync }}$. Statement $s$ is recorded and set as the upper bound of event $e_{a s y n c}$ and $e_{a s y n c}$ is restored to List. Finally, schedule spaces for all async events in List are recorded.

\subsection{Scheduling Events}

Schedule space of each async event in the event trace List is identified in the previous steps. Next, we explore event orders during test execution. An async event $e_{\text {async }}$ can be simply represented by a triplet $\langle i d, n, m\rangle$, where $n$ and $m$ are bounds of the scheduling space of $e_{\text {as } y n c}$. Specifically, $n$ is the index of the statement in the

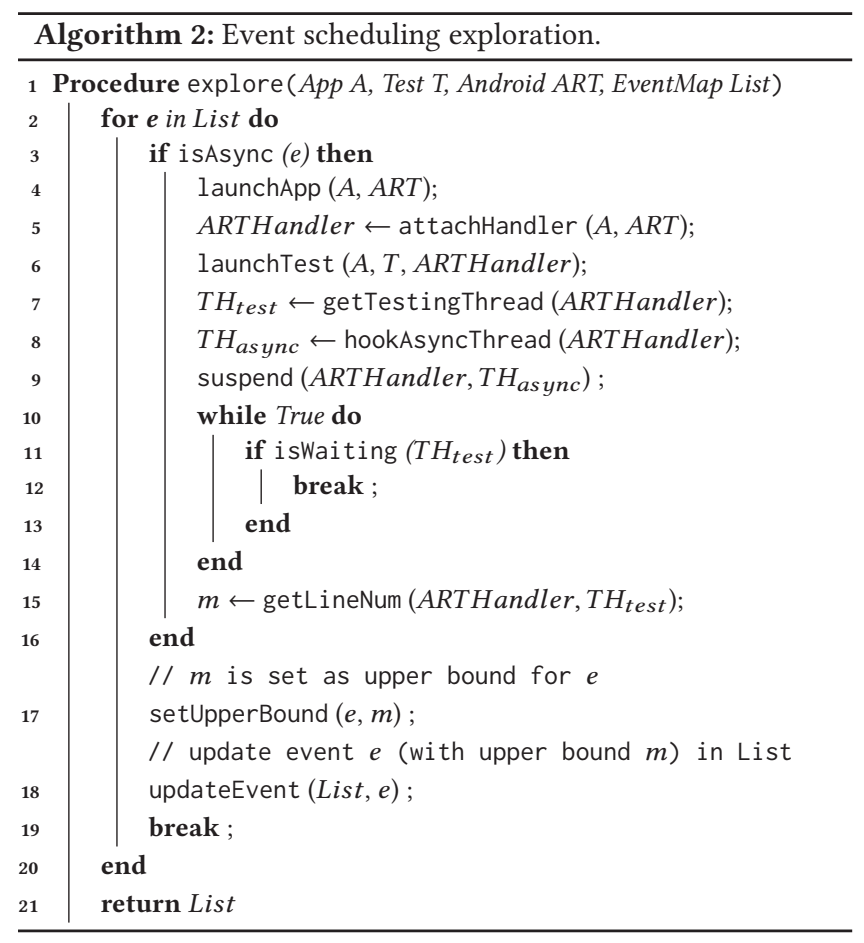

test that triggers $e_{a s y n c}$, and $m$ is the index of the statement that triggers the upper bound event of $e_{\text {as ync }}$.

Similar to scheduling space identification, we can schedule $e_{\text {as } y n c}$ by operating threads. We first hook event $e_{a s y n c}$ after the test is launched and suspend the thread that posts $e_{\text {as } y n c}$. Then, we free the testing thread and monitor whether the statement being executed is statement $m$. Once statement $m$ is reached, we suspend the testing thread and free the suspended thread to post $e_{\text {async }}$. After the async thread is terminated or idle, i.e., event $e_{a s y n c}$ has been posted, we free the testing thread. In such a way, event $e_{\text {as } y n c}$ can be executed before statement $m$. In next test run, we schedule $e_{a s y n c}$ to be executed prior to statement $m-1$, until all statements between $n$ and $m$ are explored. This procedure is repeated for each async event in List.

\section{IMPLEMENTATION}

Our system is implemented in Scala and runs on a computer that connects a physical Android device or an emulator. Unlike existing techniques, it requires no instrumentation on apps or the Android framework and can be adapted to different versions of Android.

Taking Control of Android Runtime. The Android framework supports running an app in the debug mode, under which the Android runtime can be fully controlled. Specifically, we connect the Android runtime via Android Debug Bridge (ADB) and use the Android debugger to execute the app under test. With the debugger's help, we can perform execution step by step and monitor the app state during thread manipulation.

Hooking Events. Android adopts the event-driven model and manages events using an event queue. Each event implements an interface method called enqueueMessage() and Android puts 


\section{Table 1: Subject Apps}

\begin{tabular}{||l|l|l|l|l||}
\hline App Name & Version & \#LOC & \#Stars & Category \\
\hline \hline Amaze File Manager & 3.4 .3 & $92.2 \mathrm{k}$ & $2.8 \mathrm{k}$ & Tools \\
\hline Youtube Extractor & 2.0 .0 & $2.7 \mathrm{k}$ & 519 & Video Players \\
\hline AntennaPod & 1.8 .1 & $102.6 \mathrm{k}$ & $2.7 \mathrm{k}$ & Music \& Audio \\
\hline Backpack Design & 2.0 .7 & $84.2 \mathrm{k}$ & 363 & Productivity \\
\hline Barista & 3.5 .0 & $67.9 \mathrm{k}$ & $1.2 \mathrm{k}$ & Productivity \\
\hline CameraView & 2.6 .1 & $40.5 \mathrm{k}$ & $2.9 \mathrm{k}$ & Photography \\
\hline Catroid & 0.9 .69 & $457.5 \mathrm{k}$ & 0 & Education \\
\hline City Localizer & 1.1 & $4 \mathrm{k}$ & 0 & Travel \& Local \\
\hline ConnectBot & 1.9 .6 & $119.7 \mathrm{k}$ & $1.4 \mathrm{k}$ & Communication \\
\hline DuckDuckGo & 5.43 .0 & $211.3 \mathrm{k}$ & $1.2 \mathrm{k}$ & Tools \\
\hline Espresso & 1.0 & $17.3 \mathrm{k}$ & $1.1 \mathrm{k}$ & Maps \& Navigation \\
\hline Firefox Focus & 8.5 .1 & $44.5 \mathrm{k}$ & $1.6 \mathrm{k}$ & Communication \\
\hline Firefox Lite & 2.18 & $1598.4 \mathrm{k}$ & 212 & Communication \\
\hline FlexBox & 2.0 .1 & $29.2 \mathrm{k}$ & $15.2 \mathrm{k}$ & Libraries \& Demo \\
\hline GnuCash & 2.4 .0 & $90.1 \mathrm{k}$ & $1 \mathrm{k}$ & Finance \\
\hline IBS FoodAnalyzer & 1.2 & $26.1 \mathrm{k}$ & 0 & Health \& Fitness \\
\hline Google I/O & 7.0 .14 & $73.5 \mathrm{k}$ & $19.6 \mathrm{k}$ & Books \& Reference \\
\hline Just Weather & 1.0 .0 & $5.9 \mathrm{k}$ & 65 & Weather \\
\hline Kaspresso & 1.1 .0 & $66.3 \mathrm{k}$ & 774 & Productivity \\
\hline KeePassDroid & 2.5 .3 & $159.7 \mathrm{k}$ & $1.2 \mathrm{k}$ & Tools \\
\hline KickMaterial & 1.0 & $79.1 \mathrm{k}$ & $1.6 \mathrm{k}$ & Crowdfunding \\
\hline KISS Launcher & 3.11 .9 & $27.2 \mathrm{k}$ & $1.4 \mathrm{k}$ & Personalisation \\
\hline MedLog & 1.0 & $65 \mathrm{k}$ & 0 & Medical \\
\hline Minimal To Do & 1.2 & $27.5 \mathrm{k}$ & $1.8 \mathrm{k}$ & Productivity \\
\hline MoneyManagerEx & 02.14 .994 & $170 \mathrm{k}$ & 265 & Finance \\
\hline My Expenses & 3.0 .7 .1 & $1835.6 \mathrm{k}$ & 248 & Finance \\
\hline NYBus & 1.0 & $6.9 \mathrm{k}$ & 272 & Transport \\
\hline Omni Notes & 6.0 .5 & $105.9 \mathrm{k}$ & $2 \mathrm{k}$ & Productivity \\
\hline OpenTasks & 1.2 .4 & $448 \mathrm{k}$ & 724 & Productivity \\
\hline ownCloud & 2.14 .2 & $333.7 \mathrm{k}$ & $2.9 \mathrm{k}$ & Productivity \\
\hline Sunflower & 0.1 .6 & $5.3 \mathrm{k}$ & $10.1 \mathrm{k}$ & Gardening \\
\hline Surveyor & 13.3 .0 & $290.4 \mathrm{k}$ & 13 & Communication \\
\hline WordPress & $14.2-\mathrm{rc}-2$ & $461.7 \mathrm{k}$ & $2.3 \mathrm{k}$ & Productivity \\
\hline
\end{tabular}

events to the queue by calling this method. We set a breakpoint at method enqueueMessage() in the debug mode. Whenever an event is generated and injected into the queue, we perform predefined operations such as suspending the event-posting thread.

Operating Threads. We leverage the Android debugger to retrieve threads running in the app under test, including the testing thread and UI thread. The Android debugger provides commands to remotely operate threads, e.g., inspecting thread status and suspending a selected thread. It also allows us to obtain the current stack frames of a running thread that are used for event identification. With the Android debugger, we can execute a testing thread step by step and observe execution at the statement level by inserting a breakpoint at each statement in the test.

\section{EVALUATION}

We empirically evaluate FlakeScanner's effectiveness in detecting flaky tests in the test suites of large-scale Android projects.

\subsection{Subject Apps}

Our technique is designed for a test that runs on the Android framework platform and checks whether it is flaky. To evaluate our technique, we need Android projects that contain such tests. Basically, there are two types of tests in Android projects: instrumented tests
Table 2: Root causes of reproduced flaky test failures and their categories.

\begin{tabular}{l|l}
\hline Categories & Description of root causes \\
\hline Async & $\begin{array}{l}\text { Do not wait or wait not enough when accessing background } \\
\text { resources or services in an async manner for tasks such as } \\
\text { image rendering, downloading from internet, and invoking } \\
\text { third party libraries. }\end{array}$ \\
\hline Event orders & $\begin{array}{l}\text { Expecting an implicit event execution order that may not } \\
\text { always occur in the test execution. The unexpected event } \\
\text { execution order leads to app misbehavior such as the soft } \\
\text { keyboard disappearing late. }\end{array}$ \\
\hline Data race & $\begin{array}{l}\text { Checking unsynchronized data because checking occurs be- } \\
\text { fore the data being updated due to the lack of thread synchro- } \\
\text { nization. }\end{array}$ \\
\hline Performing app-state-sensitive operations on the incorrect \\
state, e.g., the testing thread attempts to operate GUI widgets \\
when the app is resumed state, which is prohibited.
\end{tabular}

that run on a physical device or Android emulator and local unit tests that run on local Java virtual machines. Thus, we need Android projects that contain instrumented tests. Unfortunately, most Android projects in existing benchmarks such as AndroTest [11], industrial app benchmark [44], and data set that is used for flaky test empirical study [42] have no instrumented tests.

Therefore, we built the first subject-suite FlakyAppRepo in which each Android project contains instrumented tests that are written by developers. As shown in Table 1, FlakyAppRepo contains 33 Android projects (majority of them are well-known, such as WordPress) and over 5000 instrumented tests from developers. We collected Android projects containing instrumented tests as follows: (1) searching popular open-source Android projects such as Firefox and manually checking their repositories to select projects with instrumented tests (intuitively, popular projects are well maintained and more likely have developer tests); (2) searching keywords such as flak, flakiness, or intermit on the Github and manually checking searched repositories to select Android projects containing instrumented tests. Furthermore, we explored these keywords in the commit history as well to include already fixed flaky tests. A few searched projects have 0 star on the Github. To achieve diversity, we did not exclude these projects and kept them in the subject-suite.

\subsection{Research Questions}

Our evaluation aims to address the following research questions.

RQ1 Can FlakeScanner detect known flaky tests that are reported by developers?

RQ2 How does FlakeScanner compare with existing techniques in terms of number of detected flaky tests?

RQ3 Can FlakeScanner be used to discover flaky tests in Android projects that were previously unknown?

\subsection{Experiment Setup}

To answer the research questions, we conduct three empirical studies on test cases that are written by developers in real world Android app projects.

7.3.1 Study 1. We first evaluate FlakeScanner's effectiveness in flaky test detection by running it on known flaky tests in Android projects and checking how many of them are marked as flaky tests by FlakeScanner. 
Table 3: Dynamic analysis based tools for detecting flaky tests or concurrency issues in Android apps.

\begin{tabular}{l|l|c|l|l}
\hline Approach & Categories & Instrumentation & Year & Reason for not selected \\
\hline RERUN & Flaky tests & No & - & $\checkmark$ \\
\hline Shaker [40] & Flaky tests & No & 2020 & $\checkmark$ \\
\hline APEChecker [16] & Async bugs & No & 2018 & publicly unavailable \\
\hline ERVA [20] & Event race & Yes & 2016 & Publicly unavailable \\
\hline EventRacer [8] & Event race & Yes & 2015 & $\checkmark$ \\
\hline AsyncDroid [24] & Async bugs & Yes & 2015 & Incompatible instrument API \\
\hline
\end{tabular}

Data Set. We selected 52 known flaky tests from FlakyAppRepo that are caused by concurrency or synchronization issues for Study 1. In Android projects, flaky tests reported by developers are marked with a dedicated annotation @FlakyTest such that flaky tests can be automatically filtered out during test execution. We identified 269 known flaky tests in FlakyAppRepo using @FlakyTest annotation. We selected known tests from them in the following manner.

Concurrency We select concurrency related flaky tests by manually analyzing (1) details about reported flaky tests such as the reason of why a test is flaky, which can be collected from detail element ${ }^{2}$ of @FlakyTest annotation; (2) commit messages when flaky tests were introduced or fixed in a subsequent version; (3) whether there exist statements in tests that are commonly involved in concurrency or synchronization execution, e.g., operations of Runnable or AsyncTask objects.

Passing We ran each selected test in our execution environment and ensured it passes in the initial run, avoiding failures due to environment setup.

Reproducing We reproduce the flaky test failure for each selected test by analyzing its commits messages and detailed description in the repository including root causes and failing scenarios. For cases lacking the description, we contacted developers to seek more insights on the failure reproduction. A test is selected if its failures is reproduced.

In the end, 52 known flaky tests were selected for the evaluation. Meanwhile, we also analyzed root causes of these flaky test failures and classified them into four categories which are shown in Table 2.

7.3.2 Study 2. To answer RQ2, we evaluate FlakeScanner and existing tools on the data set used in Study 1. We reviewed most recent works on flaky tests or event race detection for Android apps and summarize them in Table 3. We selected the following approaches for comparison.

- RERUN. In practice, developers often run a test many times to check whether the test is flaky. Similarly, we run a test 100 times and check whether the flaky test failure manifests during execution. We call the approach 100RUN and take it as our baseline.

- Shaker [40] is the most recently reported state-of-the-art technique for detecting concurrency-related flaky tests in Android apps. Shaker attempts to manifest a flaky test failure by adding noises in the execution environment to affect event execution order, e.g., changing CPU workload.

- EventRacer [8] is a the-state-of-art dynamic technique of detecting event races in Android apps, which infers races

\footnotetext{
${ }^{2}$ https://developer.android.com/reference/androidx/test/filters/FlakyTest
}

by analyzing runtime traces produced by an instrumented Android framework.

7.3.3 Study 3. To answer RQ3, we run FlakeScanner on tests in FlakyAppRepo without @FlakyTest annotation, i.e., tests that are not reported as flaky tests. We first run these tests in our execution environment and exclude tests that fail, then feed passing tests to FlakeScanner and report tests that are labeled as flaky tests by FlakeScanner.

Effect of Debug Mode. FlakeScanner runs apps in the debug mode. The debug mode environment may affect flaky test detection by impacting the Android event dispatching mechanism or increasing execution workload. According to the official Android document ${ }^{3}$, the difference between normal execution mode and debug mode is that the Android virtual machine (VM) loads an additional Android Runtime Tooling Interface (ARTTI) plugin that exposes runtime internals (e.g., variable values). The ARTTI plugin runs as a VMlevel component and it is transparent to event dispatching that occurs in the app's main thread, i.e., running apps in debug mode does not impact the mechanism of event dispatching.

However, running the ARTTI plugin and accessing runtime internals in the execution could increase the workload of the VM. Due to the workload change, a test can manifest the flaky test failure. To rule out such cases, for each test in our study, we pre-execute it to ensure the test passes in the debug mode environment. To perform end-to-end comparison in Study 2, we run 100RUN and Shaker in the debug mode environment as well.

Execution Environment. We conducted experiments on a physical machine with 64 GB RAM and a 56 cores Intel(R) Xeon(R) E5-2660 v4 CPU, running a 64-bit Ubuntu 16.04 operating system. Each execution instance runs in a Docker container to minimize the potential inference between running instances. App under test runs on an Android 9 (x86) emulator. One execution instance is for one test case for which the Android emulator is initialized to a fresh state at the beginning to provide a clean testing environment.

\subsection{RQ1: Efficacy}

Table 4 shows results on each known flaky test for study 1 . The first column indicates known test Ids, the second column shows app names and testing frameworks used in apps, and the third column indicates test method names. Column "\#Event" indicates the number of events observed by FlakeScanner during detection. "\#Run" indicates the number of test runs in the event order exploration. Column "Time" reports the time that is used to detect a flaky test. Column "Succ" indicates whether the test is identified as a flaky test by FlakeScanner. "-" indicates that a test is unable to be executed due to library compatibility issues. Firefox-Lite marked with "*” (rows 39-40) is a previous version (commit:465739510e). Note that some test names in the table are shortened for readability.

Results. FlakeScanner successfully detected 45 out of 52 known flaky tests that are from 10 Android projects (including a different version of Firefox Lite). On average, FlakeScanner detected a flaky test in 101 seconds. The maximum detection time is for test 20 from app AntennaPod (691 seconds). The minimum detection time is for

\footnotetext{
${ }^{3}$ https://source.android.com/devices/tech/dalvik/art-ti
} 
Table 4: Results on known flaky tests by FlakeScanner, Shaker and 100RUN.

\begin{tabular}{|c|c|c|c|c|c|c|c|c|c|c|}
\hline \multirow[b]{2}{*}{ Id } & \multirow{2}{*}{$\begin{array}{l}\text { App: } \\
\text { Framework }\end{array}$} & \multirow{2}{*}{ Method name } & \multicolumn{4}{|c|}{ FlakeScanner } & \multicolumn{2}{|c|}{ Shaker } & \multicolumn{2}{|c|}{ 100Run } \\
\hline & & & \#Events & \#Run & Time(s) & Succ & Time(s) & Succ & Time(s) & Succ \\
\hline 1 & \multirow{4}{*}{$\begin{array}{l}\text { Surveyor: } \\
\text { Espresso }\end{array}$} & capture & 58 & 2 & 48 & $\checkmark$ & 4301.76 & \multirow{4}{*}{$\checkmark$} & 80 & \\
\hline 2 & & twoQuestions & 104 & 2 & 48 & $\checkmark$ & 2639.1 & & 1236 & \\
\hline 3 & & multimedia & 233 & 8 & 542 & $\checkmark$ & 1607.28 & & 486 & \\
\hline 4 & & contactDetails & 104 & 2 & 48 & $\checkmark$ & 2819.93 & & 1300 & \\
\hline 5 & \multirow{4}{*}{$\begin{array}{l}\text { Youtube } \\
\text { Extractor: } \\
\text { JUnit4 }\end{array}$} & testEncipheredVideo & 4 & 2 & 24 & $\checkmark$ & 112.136 & \multirow[t]{4}{*}{$\checkmark$} & 9 & \multirow[t]{4}{*}{$\checkmark$} \\
\hline 6 & & testUnembeddable & 7 & 3 & 22 & & 151.783 & & 103 & \\
\hline 7 & & testAgeRestrictVideo & 5 & 3 & 12 & & 103.136 & & 78 & \\
\hline 8 & & testUsualVideo & 5 & 3 & 14 & & 107.094 & & 77 & \\
\hline 9 & \multirow{5}{*}{$\begin{array}{l}\text { MyExpenses: } \\
\text { Espresso }\end{array}$} & testScenarioForBug5b.. & 861 & 2 & 100 & $\checkmark$ & 583.922 & \multirow[t]{5}{*}{$\checkmark$} & 704 & \\
\hline 10 & & editCommandKeeps.. & 101 & 2 & 54 & $\checkmark$ & 929.601 & & 857 & \\
\hline 11 & & cloneCommandIncreases.. & - & - & - & - & 949.274 & & 879 & \\
\hline 12 & & changeOfFractionDigits.. & 991 & 2 & 170 & $\checkmark$ & 672.984 & & 693 & \\
\hline 13 & & changeOfFractionDigitsWith.. & 991 & 2 & 174 & $\checkmark$ & 589.684 & & 637 & \\
\hline 14 & \multirow{4}{*}{$\begin{array}{l}\text { Firefox Lite: } \\
\text { Espresso }\end{array}$} & saveImageThenDelete.. & 627 & 5 & 352 & $\checkmark$ & 1954.07 & \multirow{2}{*}{$\checkmark$} & 1088 & \multirow[t]{4}{*}{$\checkmark$} \\
\hline 15 & & dismissMenu & 165 & 2 & 76 & $\checkmark$ & 1381 & & 742 & \\
\hline 16 & & turnOnTurboMode.. & 89 & 2 & 40 & $\checkmark$ & 926.728 & $\checkmark$ & 5 & \\
\hline 17 & & changeDisplayLang & 189 & 3 & 72 & $\checkmark$ & 3056.51 & $\checkmark$ & 680 & \\
\hline 18 & & testGoToPreferences & 107 & 3 & 56 & $\checkmark$ & 418.265 & & 4 & $\checkmark$ \\
\hline 19 & & testClickNavDrawer & 132 & 2 & 60 & $\checkmark$ & 2279.01 & $\checkmark$ & 5 & $\checkmark$ \\
\hline 20 & & PlaybackSonicTest\#..On.. & 177 & 10 & 691 & $\checkmark$ & 3243.652 & $\checkmark$ & 1128 & \\
\hline 21 & Robotium & PlaybackSonicTest\#..Off.. & 176 & 6 & 495 & $\checkmark$ & 1233.66 & & 1231 & \\
\hline 22 & & PlaybackTest\#..Off..Episodes & 175 & 2 & 142 & $\checkmark$ & 1251.95 & & 1237 & \\
\hline 23 & & PlaybackTest\#..On..Episodes & 162 & 2 & 140 & $\checkmark$ & 1071.3 & & 1128 & \\
\hline 24 & & testScrollToPosition..row & 219 & 6 & 306 & & 416.673 & & 837 & \\
\hline 25 & & testAddViewHolders.. & 46 & 2 & 17 & $\checkmark$ & 142.923 & & 196 & \\
\hline 26 & & testChangeAttributes.. & 23 & 2 & 20 & $\checkmark$ & 102.37 & $\checkmark$ & 3 & $\checkmark$ \\
\hline 27 & & testMinHeight..minHeight & 58 & 7 & 112 & $\checkmark$ & 105.134 & & 176 & \\
\hline 28 & & testJustifyContent..views & 58 & 2 & 40 & $\checkmark$ & 110.923 & & 168 & \\
\hline 29 & & testJustifyContent_center & 58 & 9 & 158 & $\checkmark$ & 211.181 & & 173 & \\
\hline 30 & Flex & testFlexWrap..column & 55 & 2 & 32 & $\checkmark$ & 107.355 & & 180 & \\
\hline 31 & Espresso & testFirstViewGone..column & 58 & 2 & 32 & $\checkmark$ & 106.491 & & 168 & \\
\hline 32 & & testChangeOrder..Params & 26 & 4 & 36 & $\checkmark$ & 102.21 & $\checkmark$ & 178 & \\
\hline 33 & & testAlignItems..column & 58 & 2 & 32 & $\checkmark$ & 107.798 & & 180 & \\
\hline 34 & & testAlignContent..column & 58 & 2 & 32 & $\checkmark$ & 107.021 & & 189 & \\
\hline 35 & & testAlignContent..column & 58 & 2 & 32 & $\checkmark$ & 105.902 & & 198 & \\
\hline 36 & & testAlignContent..Padding & 56 & 2 & 42 & $\checkmark$ & 107.256 & & 193 & \\
\hline 37 & & testFlexLines..row & 74 & 3 & 32 & $\checkmark$ & 246.254 & & 344 & \\
\hline 38 & & testFlexLines..column & 69 & 2 & 36 & $\checkmark$ & 221.768 & & 366 & \\
\hline 39 & Firefox Lite $(\star)$ : & browsingWebsite.. & 642 & 2 & 94 & $\checkmark$ & 2192.65 & $\checkmark$ & 159 & $\checkmark$ \\
\hline 40 & Espresso & saveImageThenDelete.. & 661 & 3 & 96 & $\checkmark$ & 1415.6 & & 1117 & \\
\hline 41 & & test_with_description & 41 & 2 & 11 & $\checkmark$ & 146.354 & & 289 & \\
\hline 42 & & test_with_title & 40 & 2 & 11 & $\checkmark$ & 150.643 & & 303 & \\
\hline 43 & & test_bottom_sheet_style & 32 & 4 & 30 & & 149.486 & & 207 & \\
\hline 44 & Dackrack: & test_alert_style & 32 & 4 & 31 & & 150.811 & & 199 & \\
\hline 45 & & screenshotTestDialog.. & 86 & 2 & 23 & $\checkmark$ & 285.117 & & 267 & \\
\hline 46 & & screenshotTestDialog & 86 & 2 & 23 & $\checkmark$ & 290.37 & & 263 & \\
\hline 47 & & test_with_buttons & 42 & 3 & 11 & $\checkmark$ & 174.781 & & 348 & \\
\hline 48 & & overflowMenuClick_byTitle & 130 & 3 & 82 & $\checkmark$ & 240.39 & $\checkmark$ & 309 & \\
\hline 49 & IUnit4 & openOverflowMenu_..Option & 58 & 2 & 21 & $\checkmark$ & 310.67 & $\checkmark$ & 323 & \\
\hline 50 & & overflowMenuClick_byId & 127 & 4 & 82 & $\checkmark$ & 280.342 & $\checkmark$ & 350 & \\
\hline 51 & Kaspresso: & CommonFlakyTest\#test & 93 & 2 & 26 & $\checkmark$ & 2232.77 & & 1896 & \\
\hline 52 & JUnit4 & UiCommonFlakyTest\#test & 94 & 5 & 295 & $\checkmark$ & 2048.74 & & 1941 & \\
\hline Avg & SUM & & 169 & 3 & 101 & 45 & 861 & 15 & 497 & 8 \\
\hline
\end{tabular}


test 41, 42, and 47 from app BackPack (11 seconds). FlakeScanner detected a flaky test within 3 test runs on average. The maximum number of test runs is for test 20 from AntennaPod (10 runs). To understand why FlakeScanner successfully detected failures within a few test runs, we inspected source code of projects. The major reason is that tests heavily use synchronization operations. For instance, test 2 from Surveyor contains 21 test statements, 15 of which use the synchronization operation provided by Espresso [2] onView(). When onView() is called in the test statement, the testing thread waits until background threads complete tasks. As designed, FlakeScanner does not perform event scheduling for those cases since async events are bounded in the execution of a test statement by the synchronization operation. Then FlakeScanner can focus on scheduling events for statements that lack synchronization operations. Moreover, FlakeScanner prioritizes exploring positions that are closer to the upper bound event, which likely triggers flaky test failures (Section 4.3). Thus, FlakeScanner could detect failures within a few test runs. The results also show FlakeScanner is a practical tool. It successfully detected flaky test failures for test 12 and 13 from MyExpenses, for which 991 events were generated in the execution. Meanwhile, FlakeScanner worked on Android projects that adopt different testing frameworks such as Espresso [2] and Robotium [3].

FlakeScanner successfully detected 45 out of 52 known flaky tests in 10 Android projects. On average, it detected a flaky test within 3 test runs.

\subsection{RQ2: Comparison with Existing Techniques}

As shown in Table 4, FlakeScanner outperforms Shaker and 100RUN in terms of both the number of detected flaky tests and the average execution time. Out of the 52 known flaky tests, FlakeScanner detected the most flaky tests (45) and is followed by Shaker (15) and 100RUN (8). For execution time, FlakeScanner detected a flaky test in 101 seconds on average, which is less than 861 seconds of Shaker and 497 seconds of 100RUN. For most of tests, 100RUN could not detect a flaky test failure in the first few runs and kept executing them until reaching 100 times, which took a longer time.

Regarding the overlap between flaky tests detected by each tool, FlakeScanner detected all the flaky tests detected by Shaker and 100RUN. But Shaker and 100RUN failed to detect the other flaky tests that were detected by FlakeScanner. The better results from FlakeScanner can be explained as follows: FlakeScanner can identify synchronization operations in the test execution and focus on scheduling events for statements that lack synchronization operations. Unexpected event execution orders that cause flaky test failures are more likely to explored by FlakeScanner.

We also evaluated EventRacer since it uses dynamic analysis to infer event race for Android apps. EventRacer reported many possible races for each test run. On average, it reported 1237 races for a test. This is because EventRacer focuses on detecting races in Android apps and does not analyze races in which testing frameworks are involved. Furthermore, EventRacer infers races by analyzing recorded traces and cannot validate whether the reported races can cause flaky test failures.
FlakeScanner outperforms Shaker and 100RUN in terms of both the number of detected flaky tests and average execution time.

\subsection{RQ3: Real-World Flaky Test Detection}

We ran 1444 passing tests from the 33 Android projects in FlakyAppRepo which are not annotated as flaky tests (these tests may or may not be flaky). Out of these 33 projects, FlakeScanner detected at least one flaky test for 19 projects, and reported 245 flaky tests in total. To validate previously unknown flaky tests that FlakeScanner detected, we randomly selected 20 out of the detected 245 flaky tests and reported them to developers. For each selected test, we manually reproduced the failure that FlakeScanner witnessed during detection and generated a detailed root-cause-analysis report, and submitted the report on the Github. At the time of writing the paper, we got responses on 15 test cases. Out of the 15 tests, 13 were confirmed as flaky tests and addressed by developers. For the other two tests, developers replied that the reported failures were not encountered yet or not reproduced at their end, without giving us further explanation. Our experience with flaky test reporting shows so far that developers are more interested in identifying which tests are flaky. Once a flaky test is detected, they appear to be more prone to removing them, rather than investigating why it is flaky.

FlakeScanner detected 245 previously unknown flaky tests in 19 widely-used Android projects. Out of the reported 20 unknown flaky tests, 13 were confirmed and addressed by developers.

\subsection{Threats to Validity}

External Validity: Threats to external validity relate to the generalizability of the experimental results. FlakeScanner is evaluated so far on 33 Android projects. Our results may not generalise beyond the 33 Android projects to which we have applied FlakeScanner. To mitigate this threat, we not only choose Android projects that are popular and well maintained but also include less popular Android projects (i.e., less stars on the Github) which were searched on the Github via keywords.

Internal Validity: Threats to internal validity concern factors in our experimental methodology that may affect our results. In Study 1 , we note that 52 concurrency or synchronization related known flaky tests are chosen by manually analyzing their related descriptions and commit messages, which might result in selection bias. Similarly, we manually analyze failures detected by each tool under evaluation and validate the results, which might introduce bias as well. To mitigate these risks, two authors of this paper independently performed the manual tasks, and cross-checked each other's results.

\subsection{Data Availability}

To facilitate future research on flaky tests, we make our prototype FlakeScanner and subject-suite FlakyAppRepo available at link https: //github.com/AndroidFlakyTest 


\section{RELATED WORK}

Flaky Test Detection. Bell et al. [6] use code coverage based differential analysis to identify flaky tests. A test is deemed flaky if it fails during regression testing and its execution does not reach any code that was recently changed by developers. Lam et al. propose iDFlakies [27], a framework for detecting order dependent flaky tests, tests that fail when run in different orders. Dutta et al. [13] develop an approach to detect random number related flaky tests, tests that fail due to difference in the sequence of random numbers generated in different runs. Shi et al. [39] propose an approach to fix order-dependent flaky tests by leveraging passing tests. Shi et al. [37] propose to rerun a test multiple times on each mutant and obtain reliable coverage results such that the effects of flaky tests on mutation testing can be mitigated. In contrast, FlakeScanner detects concurrency or synchronization related flaky tests in Android projects by exploring feasible event execution orders.

Event Race Detection. Another branch of works that are close to ours is event race detection [8, 18-21, 31-33, 35, 36, 45]. Instead of detecting flaky tests, these works leverage dynamic and static analysis to detect event races. For instance, ER Catcher [35], DROIDRACER [32], EventRacer [8], CAFA [19], and nAdroid [18] capture happens-before-relation among events and infer possible event races. In addition, Ozkan et al. [24] propose to detect asynchronous bugs by exploring different execution orders of event handlers in Android apps. SARD [46] leverages happens-before analysis to detect use-after-free issues in Android apps. These techniques have the potential to be applicable for flaky test detection, but face challenges to capture complete and precise happens-before relations when a test is executed by a testing framework. Many false positives can be reported by event-race detectors due to incomplete happen-before relations being compute. In contrast, FlakeScanner performs a system-level dynamic analysis to capture precise event dependencies to avoid such false positives.

Empirical Studies on Flaky Tests. Multiple studies [14, 26, 30, 42] confirm concurrency as the major cause of flaky tests. Luo et al. [30] performed an empirical analysis of flaky tests in 51 open-source projects. They identified Concurrency and Async wait as the most common cause of flaky tests. They pointed out that the majority of these cases arose because they do not wait for external resources. Finally, they described the common fixing strategies the developers use to fix flaky tests. In a separate study, Eck et al.[14] surveyed 21 professional developers to classify 200 flaky tests they fixed. They identified four unreported causes of flaky tests, which are also considered difficult to fix. Thorve et al. [42] conducted an empirical study of flaky tests in Android apps. They searched 1000 projects for the commits related to flakiness and found only 77 relevant commits from 29 projects. They found $36 \%$ of commits occurred due to concurrency related issues. Fan et al. [16] proposed a hybrid approach towards manifesting asynchronous bugs in Android apps with fault patterns.

Concurrency Bug Detection. There have been several testing based approaches [10,12, 22, 23, 28, 47] to identify concurrency related bugs. Maple [47] proposed a coverage-driven approach to expose untested thread interleavings. Letko [28] proposed a combination of testing and dynamic analysis with metaheuuristic techniques.
Choudhary et al. [10] presented a coverage-guided approach for generating concurrency tests to detect bugs in thread-safe classes. Multiple related works [5, 7, 9, 15, 29, 43] manipulated event orders to control non-determinism in multi-threaded programs. Liu et al. [29] proposed a deterministic multithreading system that replaces pthreads library in $\mathrm{C} / \mathrm{C}++$ apps. Emmi et al. [15] proposed a search prioritization strategy to discover concurrency bugs. They add non-determinism to deterministic schedulers by delaying their next-scheduled task. Adamsen et al. [5] presented an automated program repair technique for event race errors in JavaScript. Given a repair policy, they controlled the event handler scheduling in the browser to avoid bad orderings.

\section{DISCUSSION}

Flaky tests pose a significant problem in validating mobile apps. In this paper, we presented an approach for detecting flaky tests via systematic event order exploration. We introduced FlakeScanner, a tool to detect flaky tests for Android apps. FlakeScanner explores the space of possible execution environments which may cause relevant threads to interleave differently. Due to the lack of a testing benchmark for flaky tests, we created the first subject-suite FlakyAppRepo that is used to study test flakiness. FlakyAppRepo contains 33 widely-used Android apps with around 2.5k stars on average in GitHub. We applied FlakeScanner to tests from FlakyAppRepo. Results show that FlakeScanner not only detected known flaky tests but also reported 245 new flaky tests. We believe that our tool and results hold out promise for tackling flaky tests, which is a significant pain point in the practice of testing.

\section{ACKNOWLEDGEMENTS}

This work was partially supported by the National Research Foundation Singapore (National Satellite of Excellence in Trustworthy Software Systems).

\section{REFERENCES}

[1] 2016. Flaky Tests at Google and How We Mitigate Them. https://testing. googleblog.com/2016/05/flaky-tests-at-google-and-how-we.html

[2] 2020. Espresso. https://developer.android.com/training/testing/espresso

[3] 2020. Robotium. https://github.com/RobotiumTech/robotium

[4] Christoffer Quist Adamsen, Gianluca Mezzetti, and Anders Møller. 2015. Systematic Execution of Android Test Suites in Adverse Conditions. In Proceedings of the 2015 International Symposium on Software Testing and Analysis. https://doi.org/10.1145/2771783.2771786

[5] C. Q. Adamsen, A. Møller, R. Karim, M. Sridharan, F. Tip, and K. Sen. 2017. Repairing Event Race Errors by Controlling Nondeterminism. In 2017 IEEE/ACM 39th International Conference on Software Engineering (ICSE). https://doi.org/10. 1109/ICSE.2017.34

[6] J. Bell, O. Legunsen, M. Hilton, L. Eloussi, T. Yung, and D. Marinov. 2018. DeFlaker: Automatically Detecting Flaky Tests. In 2018 IEEE/ACM 40th International Conference on Software Engineering (ICSE). https://doi.org/10.1145/3180155.3180164

[7] Tom Bergan, Luis Ceze, and Dan Grossman. 2013. Input-covering schedules for multithreaded programs. (2013). https://doi.org/10.1145/2509136.2509508

[8] Pavol Bielik, Veselin Raychev, and Martin Vechev. 2015. Scalable Race Detection for Android Applications. (2015). https://doi.org/10.1145/2814270.2814303

[9] Ahmed Bouajjani, Michael Emmi, Constantin Enea, Burcu Kulahcioglu Ozkan, and Serdar Tasiran. 2017. Verifying Robustness of Event-Driven Asynchronous Programs Against Concurrency. https://doi.org/10.1007/978-3-662-54434-1_7

[10] Ankit Choudhary, Shan Lu, and Michael Pradel. 2017. Efficient Detection of Thread Safety Violations via Coverage-Guided Generation of Concurrent Tests. In IEEE/ACM International Conference on Software Engineering. https://doi.org/ 10.1109/ICSE.2017.32

[11] Shauvik Roy Choudhary, Alessandra Gorla, and Alessandro Orso. 2015. Automated Test Input Generation for Android: Are We There Yet?. In Proceedings of the 
2015 30th IEEE/ACM International Conference on Automated Software Engineering (ASE). https://doi.org/10.1109/ASE.2015.89

[12] James Davis, Arun Thekumparampil, and Dongyoon Lee. 2017. Node.fz: Fuzzing the Server-Side Event-Driven Architecture. In European Conference on Computer Systems. https://doi.org/10.1145/3064176.3064188

[13] Saikat Dutta, August Shi, Rutvik Choudhary, Zhekun Zhang, Aryaman Jain, and Sasa Misailovic. 2020. Detecting Flaky Tests in Probabilistic and Machine Learning Applications. In Proceedings of the 29th ACM SIGSOFT International Symposium on Software Testing and Analysis. https://doi.org/10.1145/3395363.3397366

[14] Moritz Eck, Fabio Palomba, Marco Castelluccio, and Alberto Bacchelli. 2019. Understanding flaky tests: the developer's perspective. In 27th ACM foint European Software Engineering Conference and Symposium on the Foundations of Software Engineering (ESEC/FSE). https://doi.org/10.1145/3338906.3338945

[15] Michael Emmi, Shaz Qadeer, and Zvonimir Rakamaric. 2011. Delay-Bounded Scheduling. In Proceedings of Symposium on Principles of Programming Languages. https://doi.org/10.1145/1926385.1926432

[16] Lingling Fan, Ting Su, Sen Chen, Guozhu Meng, Yang Liu, Lihua Xu, and Geguang Pu. 2018. Efficiently Manifesting Asynchronous Programming Errors in Android Apps. In Proceedings of the 33rd ACM/IEEE International Conference on Automated Software Engineering. https://doi.org/10.1145/3238147.3238170

[17] Martin Flower. 2020. Eradicating non-determinism in tests. https://martinfowler com/articles/nonDeterminism.html

[18] Xinwei Fu, Dongyoon Lee, and Changhee Jung. 2018. nAdroid: statically detecting ordering violations in Android applications. In Proceedings of the 2018 International Symposium on Code Generation and Optimization. https: //doi.org/10.1145/3168829

[19] Chun-Hung Hsiao, Jie Yu, Satish Narayanasamy, Ziyun Kong, Cristiano Pereira Gilles Pokam, Peter Chen, and Jason Flinn. 2014. Race Detection for Event-Driven Mobile Applications. (2014). https://doi.org/10.1145/2594291.2594330

[20] Yongjian Hu, Iulian Neamtiu, and Arash Alavi. 2016. Automatically verifying and reproducing event-based races in Android apps. In International Symposium on Software Testing and Analysis. https://doi.org/10.1145/2931037.2931069

[21] Jeff Huang and Arun K. Rajagopalan. 2016. Precise and Maximal Race Detection from Incomplete Traces. In Proceedings of the 2016 ACM SIGPLAN International Conference on Object-Oriented Programming, Systems, Languages, and Applications. https://doi.org/10.1145/2983990.2984024

[22] Casey Klein, Matthew Flatt, and Robert Findler. 2010. Random Testing for HigherOrder, Stateful Programs. In Proceedings of the Conference on Object-Oriented Programming Systems, Languages, and Applications. https://doi.org/10.1145/ 1869459.1869505

[23] Bohuslav Krena, Zdenek Letko, Tomas Vojnar, and Shmuel Ur. 2010. A platform for search-based testing of concurrent software. In International Workshop on Parallel and Distributed Systems: Testing, Analysis, and Debugging. https://doi. org/10.1145/1866210.1866215

[24] Burcu Kulahcioglu Ozkan, Michael Emmi, and Serdar Tasiran. 2015. Systematic Asynchrony Bug Exploration for Android Apps. In International Conference on Computer Aided Verification. https://doi.org/10.1007/978-3-319-21690-4_28

[25] Wing Lam, Patrice Godefroid, Suman Nath, Anirudh Santhiar, and Suresh Thummalapenta. 2019. Root causing flaky tests in a large-scale industrial setting. In Proceedings of the 28th ACM SIGSOFT International Symposium on Software Testing and Analysis. https://doi.org/10.1145/3293882.3330570

[26] Wing Lam, Kivanc Muslu, Hitesh Sajnani, and Suresh Thummalapenta. 2020. A Study on the Lifecycle of Flaky Tests. In 42nd International Conference on Software Engineering. https://doi.org/10.1145/3377811.3381749

[27] W. Lam, R. Oei, A. Shi, D. Marinov, and T. Xie. 2019. iDFlakies: A Framework for Detecting and Partially Classifying Flaky Tests. In 12th IEEE Conference on Software Testing, Validation and Verification. https://doi.org/10.1145/3238147. 3240465

[28] Zdeněk Letko. 2013. Analysis and Testing of Concurrent Programs. Information Sciences and Technologies Bulletin of the ACM Slovakia (2013).

[29] Tongping Liu, Charlie Curtsinger, and Emery Berger. 2011. Dthreads: Efficient deterministic multithreading. In Proceedings of the 23rd ACM Symposium on Operating Systems Principles. https://doi.org/10.1145/2043556.2043587

[30] Qingzhou Luo, Farah Hariri, Lamyaa Eloussi, and Darko Marinov. 2014. An empirical analysis of flaky tests. In International Symposium on Foundations of Software Engineering (FSE). https://doi.org/10.1145/2635868.2635920

[31] Pallavi Maiya and Aditya Kanade. 2017. Efficient Computation of Happens-before Relation for Event-Driven Programs. In Proceedings of the 26th ACM SIGSOFT International Symposium on Software Testing and Analysis. https://doi.org/10.
$1145 / 3092703.3092733$

[32] Pallavi Maiya, Aditya Kanade, and Rupak Majumdar. 2014. Race Detection for Android Applications. In Proceedings of the 35th ACM SIGPLAN Conference on Programming Language Design and Implementation. https://doi.org/10.1145/ 2594291.2594311

[33] Arun K. Rajagopalan and Jeff Huang. 2015. RDIT: Race Detection from Incomplete Traces. In Proceedings of the 2015 10th foint Meeting on Foundations of Software Engineering. https://doi.org/10.1145/2786805.2803209

[34] Alan Romano, Zihe Song, Sampath Grandhi, Wei Yang, and Weihang Wang. 2021. An Empirical Analysis of UI-based Flaky Tests. In IEEE/ACM International Conference on Software Engineering. https://doi.org/10.1109/ICSE43902.2021. 00141

[35] Navid Salehnamadi, Abdulaziz Alshayban, Iftekhar Ahmed, and Sam Malek. 2020. ER Catcher: A Static Analysis Framework for Accurate and Scalable EventRace Detection in Android. In Proceedings of the 35th IEEE/ACM International Conference on Automated Software Engineering. https://doi.org/10.1145/3324884. 3416639

[36] Anirudh Santhiar, Shalini Kaleeswaran, and Aditya Kanade. 2016. Efficient Race Detection in the Presence of Programmatic Event Loops. In Proceedings of the 25th International Symposium on Software Testing and Analysis. https: //doi.org/10.1145/2931037.2931068

[37] August Shi, Jonathan Bell, and Darko Marinov. 2019. Mitigating the effects of flaky tests on mutation testing. In Proceedings of the 28th ACM SIGSOFT International Symposium on Software Testing and Analysis. https://doi.org/10.1145/3293882. 3330568

[38] A. Shi, A. Gyori, O. Legunsen, and D. Marinov. 2016. Detecting Assumptions on Deterministic Implementations of Non-deterministic Specifications. In 2016 IEEE International Conference on Software Testing, Verification and Validation. https://doi.org/10.1109/ICST.2016.40

[39] August Shi, Wing Lam, Reed Oei, Tao Xie, and Darko Marinov. 2019. iFixFlakies: a framework for automatically fixing order-dependent flaky tests. In Proceedings of the 27th ACM foint Meeting on European Software Engineering Conference and Symposium on the Foundations of Software Engineering (ESEC-FSE). https: //doi.org/10.1145/3338906.3338925

[40] Denini Silva, Leopoldo Teixeira, and Marcelo d'Amorim. 2020. Shake It! Detecting Flaky Tests Caused by Concurrency with Shaker. In IEEE International Conference on Software Maintenance and Evolution. https://doi.org/10.1109/ICSME46990. 2020.00037

[41] Valerio Terragni, Pasquale Salza, and Filomena Ferrucci. 2020. A Container-Based Infrastructure for Fuzzy-Driven Root Causing of Flaky Tests. In Proceedings of the ACM/IEEE 42nd International Conference on Software Engineering: New Ideas and Emerging Results. https://doi.org/10.1145/3377816.3381742

[42] Swapna Thorve, Chandani Sreshtha, and Na Meng. 2018. An Empirical Study of Flaky Tests in Android Apps. In International Conference on Software Maintenance and Evolution. https://doi.org/10.1109/ICSME.2018.00062

[43] Ermenegildo Tomasco, Omar Inverso, Bernd Fischer, Salvatore La Torre, and Gennaro Parlato. 2015. Verifying Concurrent Programs by Memory Unwinding. In 21st International Conference on Tools and Algorithms for the Construction and Analysis of Systems. https://doi.org/10.1007/978-3-662-46681-0 52

[44] Wenyu Wang, Dengfeng Li, Wei Yang, Yurui Cao, Zhenwen Žhang, Yuetang Deng, and Tao Xie. 2018. An Empirical Study of Android Test Generation Tools in Industrial Cases. In Proceedings of the 33rd ACM/IEEE International Conference on Automated Software Engineering.

[45] D. Wu, J. Liu, Y. Sui, S. Chen, and J. Xue. 2019. Precise Static Happens-Before Analysis for Detecting UAF Order Violations in Android. In 2019 12th IEEE Conference on Software Testing, Validation and Verification (ICST). https://doi. org/10.1109/ICST.2019.00035

[46] Diyu Wu, Jie Liu, Yulei Sui, Shiping Chen, and Jingling Xue. 2019. Precise Static Happens-Before Analysis for Detecting UAF Order Violations in Android. In 2019 12th IEEE Conference on Software Testing, Validation and Verification (ICST). https://doi.org/10.1109/ICST.2019.00035

[47] Jie Yu, Satish Narayanasamy, Cristiano Pereira, and Gilles Pokam. 2012. Maple: A Coverage-Driven Testing Tool for Multithreaded Programs. In Proceedings of the Conference on Object-Oriented Programming Systems, Languages, and Applications. https://doi.org/10.1145/2384616.2384651

[48] Sai Zhang, Darioush Jalali, Jochen Wuttke, Kıvanç Mușlu, Wing Lam, Michael D. Ernst, and David Notkin. 2014. Empirically revisiting the test independence assumption. In Proceedings of the 2014 International Symposium on Software Testing and Analysis. https://doi.org/10.1145/2610384.2610404 